\title{
Eye Development
}

National Cancer Institute

\section{Source}

National Cancer Institute. Eye Development. NCI Thesaurus. Code C19413.

Human Eye Development involves bilateral outpouchings of optic vesicles, from the neural tube, that each form optic cups. By cell division, migration, differentiation, and synaptogenesis, the inner surface becomes the multi-layer neuroepithelial retina; the outer monolayer becomes the retinal pigment epithelium. Surface membranes cover the eye and develop into the lens, iris and cornea. 